\title{
ROLE OF TRIPLE PHASE MDCT IN THE CHARACTERISATION OF LIVER LESIONS
}

\author{
Bharathi Selvam Sengodan', Senthil Kumar Aiyappan², Bulabai Karpagam³
}

${ }^{1}$ Senior Resident, Department of Radiology, SRM Medical College Hospital and Research Centre, Kancheepuram, Tamilnadu. ${ }^{2}$ Associate Professor, Department of Radiology, SRM Medical College Hospital and Research Centre, Kancheepuram, Tamilnadu. ${ }^{3}$ Professor, Department of Radiology, SRM Medical College Hospital and Research Centre, Kancheepuram, Tamilnadu.

\section{ABSTRACT}

\section{BACKGROUND}

MDCT is a very useful investigative technique for all liver pathologies. The conspicuity of a liver lesion on CT depends on the attenuation difference between the lesion and the normal liver. The difference in blood supply results in different enhancement patterns between various liver tumours in the various phases of contrast enhancement. Intravenous contrast is needed to increase the conspicuity of lesions. Multidetector computed tomography with plain, arterial, portal venous and delayed phase is very u seful in identifying and characterising various liver lesions depending on their vascular nature. In our study, we evaluated the role of Multidetector computed tomography in the characterisation of liver lesions in patients presenting to our hospital.

\section{MATERIALS AND METHODS}

The present study was conducted on 40 patients with known or suspected liver lesions between September 2015 to September 2016 in the Department of Radiodiagnosis, in coordination with the Department of General Surgery, SRM Medical College Hospital and Research Centre, Kancheepuram, Tamilnadu. Triple phase multidetector CT was done for all patients with focal liver lesions and the results are correlated with histopathology. The percentage of accuracy in diagnosis of liver lesions by MDCT was calculated from the number of correctly and wrongly diagnosed cases.

\section{RESULTS}

The age distribution of the patients was relatively wide, ranging from 35-75 years. The commonest symptom in the cases was pain in the abdomen (42\%), followed by abdominal distension (25\%), jaundice (17\%), vomiting (8\%), and fever (8\%). $80 \%$ of the cases showed multiple liver lesions and $20 \%$ cases showed single lesion. The accuracy of MDCT in characterisation of liver lesions was found to be 97.5 percentage.

\section{CONCLUSION}

MDCT with its triple phase protocol is a very good imaging modality for the characterisation of the liver lesions with a diagnostic accuracy of $97.5 \%$ as also observed in this study.

\section{KEYWORDS}

Liver, MDCT, Triple Phase, Haemangioma, Metastasis.

HOW TO CITE THIS ARTICLE: Sengodan BS, Aiyappan SK, Karpagam B. Role of triple phase MDCT in the characterisation of liver lesions. J. Evolution Med. Dent. Sci. 2017;6(24):2003-2008, DOI: 10.14260/Jemds/2017/438

\section{BACKGROUND}

The liver, due to its major function of detoxification and its rich blood supply by hepatic artery and portal vein becomes prone to various diseases. The primary objective in imaging the liver is to distinguish benign from metastatic and primary malignant lesion. ${ }^{1}$ The practice for liver imaging has undergone a significant change over the past two decades. Many different techniques are available for imaging the liver. They include the long established X-rays, the second generation tools of arteriography, radionuclide scanning. Since the 1970s, the primary radiological evaluation of the liver using nuclear scintigraphy and angiography has given way to computed tomography (CT), ultrasonography, and magnetic resonance imaging (MRI). ${ }^{2-7}$

Financial or Other, Competing Interest: None.

Submission 25-01-2017, Peer Review 10-03-2017,

Acceptance 16-03-2017, Published 23-03-2017.

Corresponding Author:

Bharathi Selvam Sengodan,

Senior Resident,

SRM Medical College Hospital and Research

Centre, Kancheepuram, Tamilnadu-603203.

E-mail: bharathiselvan.s@gmail.com

DOI: $10.14260 /$ jemds $/ 2017 / 438$

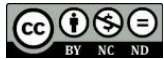

Angiography is now more applicable (as digital subtraction angiography or DSA) to interventional techniques and therapy.

Multidetector CT (MDCT) is very useful investigative technique for all liver pathologies. The conspicuity of a liver lesion on CT depends on the attenuation difference between the lesion and the normal liver. On a non-enhanced CT-scan (NCCT), liver tumours usually are not visible, because the inherent contrast between tumour tissue and the surrounding liver parenchyma is too low. Only a minority of tumours that contain calcifications, cystic components, fat or haemorrhage and will be detected. $80 \%$ of normal liver parenchyma is supplied by the portal vein and $20 \%$ by the hepatic artery. The difference in blood supply results in different enhancement patterns between various liver tumours in the various phases of contrast enhancement. ${ }^{2}$ Intravenous contrast is needed to increase the conspicuity of lesions. The development of Multidetector row CT technology has helped CT to continue to excel in its already established indication i.e., hepatic lesion detection and characterisation and to add new clinical indication i.e., CT angiography for preprocedure mapping and liver perfusion. With MDCT there is faster scanning and in liver imaging, faster scanning decreases respiration artefacts and improves multiphase 
imaging. Dynamic CT with arterial and portal venous phase imaging is now considered to be the primary way of approach for the diagnosis of liver lesions. Multidetector computed tomography with plain, arterial, portal venous and delayed phase is very useful in identifying and characterising various liver lesions depending on their vascular nature. In our study, we evaluated the role of Multidetector computed tomography in the characterisation of liver lesions in patients presenting to our hospital.

\section{MATERIALS AND METHODS}

The present study was conducted on 40 patients presenting to the Department of Radiodiagnosis and Department of General Surgery, SRM Medical College Hospital and Research Centre, Kancheepuram, Tamilnadu for evaluation of known or suspected liver lesions from September 2015 to September 2016. All basic examinations were performed and written informed consent was taken prior to any intervention. The aim of the study was to study the enhancement pattern and characterisation of various liver lesions in multidetector computed tomography. This was a descriptive study and the patients presenting to the OPD of the Department of Surgery and Department of Radiodiagnosis with known and suspected liver lesions was selected for the study. The patients with previous known allergy to medications, reduced renal function with elevated blood creatinine levels and history of intervention in the form of previous surgery to the liver lesion were excluded from the study. The exact plan of the study was individualised for each case. When performing the study the patient was made to lie on the scan table and imaged using a GE Optima 128-slice CT with non-contrast and contrast enhanced study using oral and intravenous contrast agents and MDCT triphasic scans with plain study, arterial phase, venous phase and delayed phase scans were taken in all the patients with breath hold. NCCT was taken from the lower thoracic level downwards up to the pelvic region covering the entire abdomen. The NCCT scans were followed by contrast enhanced multiphase imaging. All patients were administered $1 \mathrm{~mL} / \mathrm{kg}$ of intravenous contrast material at an injection rate of 4-6 mL/sec. The phases were obtained using SmartPrep technique. From this data, the plain and contrast enhanced axial scans, multiplanar (coronal, sagittal, oblique \& curved), MIP (Maximum intensity projection) and MinIP (minimum intensity projection) were viewed. Based on the clinical symptoms and imaging features all the 40 cases were given a specific radiological diagnosis. The patients were followed up and final diagnosis was confirmed by histopathological analysis with FNAC/biopsy. By comparing the imaging diagnosis with corresponding histopathological final diagnosis, the diagnostic accuracy of the MDCT on the liver lesion was ascertained.

\section{RESULTS}

The present study was conducted on 40 patients in the Department of Radiodiagnosis, in coordination with the Department of General Surgery. The age distribution of the patients was relatively wide, ranging from 35-75 years [Table-1]. The maximum number of cases were seen among the 51-60 years group (32\%). The maximum age of the patients included in the study was 75 years and the minimum age included was 35 years. Of the 40 patients included in the study, 21 patients were male $(52 \%)$ and 19 patients were female (48\%). The commonest symptom in the cases was pain in the abdomen (42\%), followed by abdominal distension (25\%), jaundice (17\%), vomiting (8\%), and fever (8\%).

\begin{tabular}{|c|c|c|}
\hline Age & Number of Cases & Percentage \% \\
\hline $21-30$ & 0 & $0 \%$ \\
\hline $31-40$ & 6 & $15 \%$ \\
\hline $41-50$ & 10 & $25 \%$ \\
\hline $51-60$ & 13 & $32 \%$ \\
\hline $61-70$ & 10 & $25 \%$ \\
\hline $71-80$ & 1 & $3 \%$ \\
\hline $81-90$ & 0 & $0 \%$ \\
\hline \multicolumn{2}{|c|}{ Table 1. Age Distribution of Cases } \\
\hline
\end{tabular}

\section{Imaging Features in MDCT}

The following imaging features were observed in the plain, arterial phase, portal venous phase and delayed phase among all the 40 cases included in the study.

\section{Number of Lesions- Single Versus Multiple}

$80 \%$ of the cases showed multiple liver lesions and $20 \%$ cases showed single lesion.

\section{Location of the Lesions}

Lesions were noted in both the lobes in $47.5 \%$ cases while $42.5 \%$ cases were seen in the right lobe and $10 \%$ cases were seen in the left lobe [Table-2].

\begin{tabular}{|c|c|c|}
\hline Location & No. of Cases & Percentage \\
\hline Right lobe & 17 & $42.5 \%$ \\
\hline Left lobe & 4 & $10 \%$ \\
\hline Both & 9 & $47.5 \%$ \\
\hline \multicolumn{2}{|c|}{ Table 2. Location of the Lesions in the Liver } \\
\hline
\end{tabular}

\section{Diagnosis based on imaging Findings in MDCT}

Of the 40 cases observed in the study, 7 cases which showed hypodensity in plain study also showed peripheral discontinuous enhancement in the arterial phase study and enhancement in portal venous phase with complete central enhancement in the delayed phase were diagnosed as haemangioma. In seven patients, the liver lesions showed hypodensity in the plain study, prominent enhancement in the arterial phase, reduced enhancement in the portal venous phase and appearing hypodense with the rest of the normal liver parenchyma in the delayed phase were diagnosed as HCC. Of the 7 cases diagnosed as HCC chronic liver disease was seen in 5 cases. 4 cases showed thrombosis of portal vein, 3 cases showed prominently enhancing intra-tumoural vessels, one case showed enhancing well-defined capsule in portal venous phase. 14 cases showed ill-defined mass lesions in liver along the gallbladder bed region with nonvisualisation of gallbladder-liver interface. The lesions showed hypodensity in the plain study and showed peripheral enhancement in the arterial and the portal venous phases with no enhancement noted in the delayed phase. Among them 8 cases showed ill-defined mass in the gallbladder fossa region with partial non-visualisation of the gallbladder, 5 cases showed wall thickening involving the adjacent gallbladder and one case showed intraluminal mass in the gallbladder. These 14 cases were diagnosed as 
infiltrating carcinoma of gallbladder. One of the case which showed hypodense lesion in the right lobe of liver on plain study showed no enhancement in arterial and portal venous phase but lesion was enhanced in delayed phase. This case also showed dilatation of the intrahepatic biliary radicles and was diagnosed as mass forming intrahepatic cholangiocarcinoma.

Multiple lesions that were hypodense and isodense in the plain study was observed in 10 cases, among them diffuse enhancement in the arterial phase was observed in 3 cases, and peripheral enhancement in portal venous phase seen in the rest of 7 cases. Among them three cases which showed enhancement in the arterial phase, two cases also showed mass lesions in the kidneys and one case showed a hypodense lesion in the head of the pancreas which was showing strong enhancement in the arterial phase. These cases were diagnosed as liver metastasis with primary mass in the kidneys and as hypervascular tumour in the pancreas. Among the 7 cases which showed peripheral enhancement in the portal venous phase, 2 cases had irregular wall thickening in the rectum and one case had ulcerated lesion in the fundus region of the stomach, hence these cases were diagnosed as carcinoma of rectum and of stomach with hepatic metastasis. Three cases showed abnormality in chest X-ray with mass in lungs and one case each had mass lesions in the right breast, hence these cases were also diagnosed as hepatic metastasis. One case showed a hypodense lesion in the right lobe of liver and showed peripheral enhancement in the arterial and the portal venous phase, the patient also had a mass lesion in the thyroid, hence the lesion was diagnosed as liver metastasis.

\section{Histopathological Analysis}

Apart from the seven cases which were diagnosed as haemangioma, 33 cases were advised tissue diagnosis and subjected to FNAC/biopsy and were followed up. Of the 7 cases diagnosed as HCC by MDCT, 6 cases were confirmed as HCC, while one case of metastatic adenocarcinoma was wrongly diagnosed as HCC. The fourteen cases which were diagnosed as infiltrating carcinoma of gallbladder and one case of cholangiocarcinoma was also confirmed with same diagnosis. All the cases reported as metastasis were confirmed as liver metastasis.

\section{Analysis of the Percentage of Accuracy}

The percentage of accuracy in diagnosis of liver lesions by MDCT was calculated from the number of correctly and wrongly diagnosed cases. The accuracy of MDCT in characterisation of liver lesions was found to be 97.5 percentage [Table-3].

\begin{tabular}{|c|c|c|}
\hline Cases & No. of Cases & Percentage \\
\hline Correctly diagnosed & 39 & $97.5 \%$ \\
\hline Incorrect diagnosis & 1 & $2.5 \%$ \\
\hline \multicolumn{2}{|c|}{ Table 3. Percentage of Accuracy in } \\
Diagnosis of Liver Lesions by MDCT \\
\hline
\end{tabular}

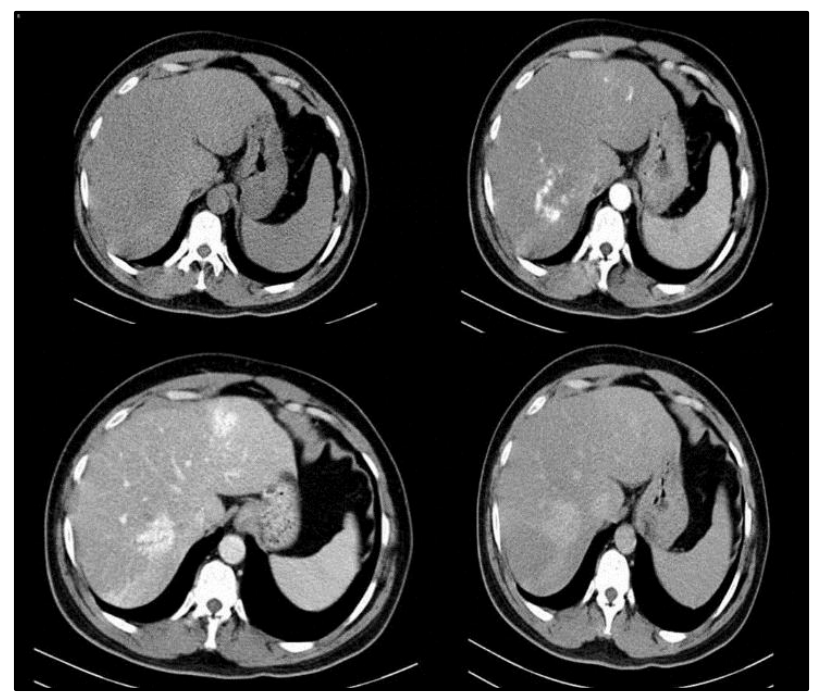

Figure 1. A Case of hemangioma, showing hypodensity in plain scan with peripheral discontinuous enhancement in arterial phase with puddling and attenuation equal to aorta in all sequences with filling in delayed phase.

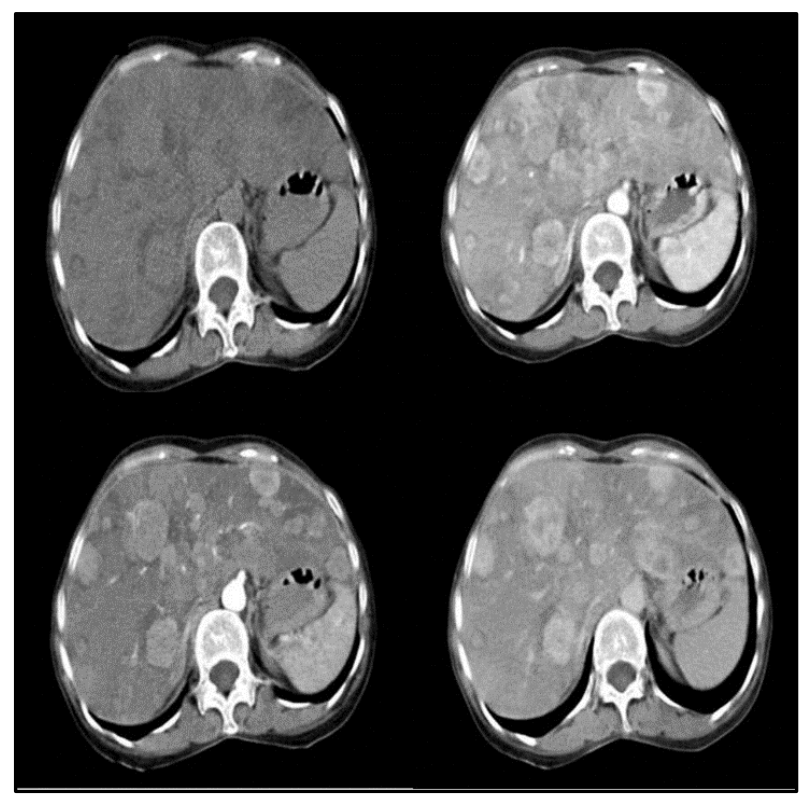

Figure 2. A case of hypervascular liver metastasis. Multiple iso /hypodense lesions in the liver showing strong enhancement predominantly in the arterial phase

\section{DISCUSSION}

The age of patients varied from 35 years to 75 years with the maximum number of cases in the age group of 51-60 years $(32 \%)$ and $52 \%(n=21)$ were male and $48 \%(n=19)$ were female. The commonest symptom in the cases was pain in the abdomen (42\%), followed by abdominal distension (25\%), jaundice $(17 \%)$, vomiting ( $8 \%)$, and fever $(8 \%)$.

In this study, 7 cases were benign and 33 cases were malignant. All the benign lesions were haemangioma and were correctly diagnosed on MDCT. Out of 33 malignant lesions, 32 were correctly diagnosed with Triple phase CT and one case was wrongly diagnosed.

The role of triple phase imaging in the detection and diagnosis of various hepatic masses is described below. 


\section{Haemangioma}

Haemangioma is the most common benign liver lesion observed in our study. According to Semelka et $\mathrm{al}^{8}{ }^{8}$ they are usually asymptomatic. Typical haemangiomas are easily diagnosed by Ultrasonography; however, difficulties arise when they are associated with cystic areas or calcifications and when they are multiple in numbers. In such instances, CT becomes imperative.

Haemangioma (Figure 1) was seen among 7 cases in our study. All the cases were female $(100 \%)$ and evaluated for symptoms not related to the haemangioma as was described by Semelka et al. ${ }^{8}$ The commonest age group noted was of 40 49 years $(n=5) .57 \%$ of cases showed single lesions and lesions were multiple in $43 \%$ cases. The lesions were noted in the right lobe of liver in 4 cases, in the both lobes in 3 cases. In all the instances, the margins of the lesions were well defined with the adjacent normal liver parenchyma.

The lesions were hypodense in $71 \%$ of the cases observed in our study $(\mathrm{n}=5)$ and isodense in $29 \%$ cases $(\mathrm{n}=2)$ in plain study and they showed early discontinuous peripheral enhancement in arterial phase with progressive centripetal filling in the delayed phase in all the cases as described by Hanafusa et al ${ }^{9}$ and Yashimata et al 10 in their studies. The peripheral enhancement of the lesions was equal to the attenuation of aorta in all the phases studied. These imaging findings are consistent with the CT imaging features of hepatic haemangioma as described by Scartarige et al. ${ }^{11}$

Quinn et al in their study could diagnose $94 \%$ of the cases as haemangioma by using the CT feature of attenuation of lesion in all the phases of study which was equal to that of aorta. In our study, we had the similar findings in all the cases of haemangioma diagnosed.12 No other additional features like lymph node enlargement or ascites was present. Three cases which had multiple lesions were suspected as metastasis on ultrasonography, but they were confirmed as haemangioma based on the imaging characteristics in MDCT study.

Hence MDCT was able to correctly diagnose all the cases of haemangioma based on MDCT characteristics and none of the cases required any further investigation to confirm the diagnosis or any active management.

\section{Hepatocellular Carcinoma}

Of the 33 cases, 7 cases were diagnosed as HCC, of which 6 cases were confirmed as HCC by biopsy and one case was wrongly diagnosed as HCC which was confirmed as metastatic adenocarcinoma of unknown primary. They were suspected based on clinical features and elevated AFP levels. The most common clinical symptom at presentation was pain in abdomen (52\%). According to Matsui et al, cirrhosis was present in up to $90 \%$ of cases with HCC 13 ; however, in our study cirrhosis was noted only in $83.3 \%$ cases.

All the lesions were hypodense on NCCT study and on CECT showed enhancement in arterial phase; however, in the portal venous phase no enhancement was seen suggestive of wash out of contrast in the portal venous phase. Two cases also showed central necrosis. Enhancement of a thin peripheral capsule in portal venous phase leading to capsular delineation was seen in one case. According to Baron et al 14 on plain study, most of HCC present as low attenuating lesions, which on arterial phase being hypervascular tumour shows intense enhancement throughout the tumour and the capsule is seen as a hypodense rim. In portal venous phase, there is rapid washout and the lesion becomes iso to hypodense compared to normal liver parenchyma. On delayed phase images, the capsule and fibrous septa may show prolonged enhancement. According to the study by Honda et al, hepatocellular carcinoma was hypervascular in $60 \%$ of the cases but in our study hypervascularity in arterial phase was noted in all the cases. ${ }^{15}$ Baron and Oliver et al had described that the tumour thrombus is one of the characteristic features of HCC whether in the portal vein or its branches, and the obstruction or invasion of portal vein or hepatic vein is seen in $30-40 \%$ of cases. In our study, we had diagnosed portal vein thrombosis in $66 \%$ of cases. MDCT with its arterial and portal venous phase study and MIP images was very advantageous in the diagnosis of HCC.

\section{Infiltrating Gallbladder Carcinoma}

Carcinoma gallbladder with infiltration of the hepatic parenchyma is one of the common liver lesions observed in the elderly. They are easily identifiable by ultrasonography; however, the vascular nature and extent are better assessed by MDCT. There were total 14 cases of Carcinoma gallbladder with hepatic infiltration in this study. The commonest morphological mode of presentation of infiltrating gallbladder carcinoma was mass lesion in the gallbladder fossa of liver in the segment IV $b$ and $V$ of liver seen in $57.1 \%$ cases $(n=8)$, followed by thickening of the gallbladder wall seen in $35.8 \%$ cases $(n=5)$ and intraluminal polypoidal growth seen in $7.1 \%$ cases $(n=1)$.

Similar findings were observed by Lt Colonel Ra George et al in their study where the mass forming type of gallbladder carcinoma was seen in $56 \%$ cases. ${ }^{16}$

Gallbladder wall calcification and gall stones were observed in 8 cases (56\%). The percentage of incidence of gall stones in our study was similar to the findings of Lt Col George et al who noted 50\% incidence of gall stones in carcinoma gallbladder. Both GB wall calcification and gall stone are implicated as an aetiological factor in the pathogenesis of gallbladder carcinoma. ${ }^{16}$

Kumar et al had described that liver is the most frequently involved organ by direct contiguous spread $(65 \%$ of cases) followed by the colon (15\%), duodenum (15\%) and pancreas (6\%). ${ }^{17}$

In our study, most of the lesions were hypodense on plain study (100\%) and showed enhancement in arterial phase and portal venous phase which was predominantly peripheral in all the cases and no delayed phase enhancement was noted. The lesions were conspicuous more in the portal venous phase than in the arterial phase. Similar CT findings were described by Lt Col George et al in their studies. ${ }^{16}$

\section{Intrahepatic Mass Forming Cholangiocarcinoma}

In this study, a 65-year-old male was diagnosed with intrahepatic mass forming cholangiocarcinoma. The patient had jaundice and hyperbilirubinaemia at presentation, Vilgrain et al have described patients with intrahepatic cholangiocarcinoma presenting with similar clinical features in their study. ${ }^{18}$ The case in our study had single lesion in right lobe with well-defined margins with intrahepatic biliary radical dilatation in right lobe of liver. Gallbladder was 
normal. The lesions were hypodense on plain study and showed no enhancement in arterial and portal venous phase but was enhanced in delayed phase. According to Asayamaa et $\mathrm{al}^{19}$ cholangiocarcinomas are usually hypo- to isoattenuating relative to the normal hepatic parenchyma at plain CT. After the intravenous administration of contrast material, most cholangiocarcinomas remain hypoattenuating during the arterial and portal venous phases and show enhancement during the delayed phase, findings that reflect their hypovascular desmoplastic stromal composition. ${ }^{19} \mathrm{We}$ also had similar MDCT findings in our case. The case in our study underwent biopsy and specimen was sent for histopathological examination which confirmed the diagnosis cholangiocarcinoma.

\section{Metastasis}

In our study, metastasis (Figure 2) were seen in 11 out of the total 40 cases $(27.5 \%)$ and it was the second largest group, majority of these cases were in the age group of 61-70 years $(n=4)$. Most of the lesions were hypodense lesions on plain study and few were isodense which was seen when they were smaller in size. 8 cases (72.7\%) showed peripheral enhancement in the arterial phase and portal venous phase with no central enhancement while 3 cases (27.3\%) showed diffuse enhancement in arterial and portal venous phases. Foley et $\mathrm{al}^{20}$ has described that MDCT appearance of hypervascular lesions are hyperenhancing on arterial and portal venous phase either homogeneous or inhomogeneous due to areas of necrosis or haemorrhage. The imaging appearance of these hypervascular tumours is distinct from rim enhancing hypovascular metastatic lesions from common primary sites, such as lung, breast, pancreas, colon, and the genital tract. Hypovascular metastasis are the most common type and these lesions on the arterial and portal venous phase may demonstrate a hyperenhancing rim that is different from that of hypervascular metastases which shows diffuse enhancement. ${ }^{21}$

In this study, we observed that hypervascular metastatic lesions were more conspicuous in the arterial phase and hypovascular metastatic lesions predominantly show only peripheral thin enhancement in the arterial phase and portal venous phase and no central enhancement.

\section{CONCLUSION}

MDCT with its triple phase protocol is a very good imaging modality for the characterisation of the liver lesions with a diagnostic accuracy of $97.5 \%$ as also observed in this study. The most common lesions observed in our study were hepatic infiltration by gallbladder carcinoma and liver metastasis which is more than the primary malignant tumours of liver. Haemangioma which is the most common benign liver lesion worldwide was the only benign lesion observed in this study. MDCT with its multiphase protocol is very useful in the characterisation of the liver lesions like haemangioma, HCC, Intrahepatic cholangiocarcinoma and metastatic lesions based on their enhancement characteristics which depend on the innate vascularity of the lesions. MDCT is useful in the evaluation of infiltrating gallbladder carcinoma, its extent, local invasion and staging.

\section{REFERENCES}

[1] Lee KH, O'Malley ME, Haider MA, et al. Triple-phase MDCT of hepatocellular carcinoma. Am J Roentgenol 2004;182(3):643-9.

[2] Hussain SM, Semelka RC. Hepatic imaging: comparison of modalities. Radiol Clin North Am 2005;43(5):92947.

[3] Hollett MD, Jeffrey RB, Nino-Murcia M, et al. Dualphase helical CT of the liver: value of arterial phase scans in the detection of small $(<$ or $=1.5 \mathrm{~cm})$ malignant hepatic neoplasms. Am J Roentgenol 1995;164(4):879-84.

[4] Miller FH, Butler RS, Hoff FL, et al. Using triphasic helical CT to detect focal hepatic lesions in patients with neoplasms. Am J Roentgenol 1998;171(3):643-9.

[5] Boll DT, Merkle EM. Liver: normal anatomy. Imaging techniques and diffuse diseases. In: Haaga CT. edr. MRI of whole body. $5^{\text {th }}$ edn. Mosby 2009; p. 166.

[6] Dodd GD. An American's guide to Couinaud's numbering system. Am J Roentgenol 1993;161(3):574-5.

[7] Prasanna PM, Fredericks SE, Winn SS, et al. Best cases from the AFIP: giant cavernous hemangioma. Radiographics 2010;30(4):1139-44.

[8] Semelka RC, Sofka CM. Hepatic hemangioma. Magn Reson Imaging Clin N Am 1997;5(2):241-53.

[9] Yamashita Y, Hatanaka Y, Yamamoto H, et al. Differential diagnosis of focal Liver lesions: role of spin-echo and contrast-enhanced dynamic MR imaging. Radiology 1994;193(1):59-65.

[10] Hanafusa K, Ohashi I, Himeno Y, et al. Hepatic hemangioma: findings with two-Phase CT. Radiology 1995;196(2):465-9.

[11] Scatarige JC, Fishman EK, Saksouk FA, et al. Computed tomography of calcified liver masses. J Computer Assisted Tomography 1983;7(1):83-9.

[12] Quinn SF, Benjamin GG. Hepatic cavernous hemangiomas: simple diagnostic sign with dynamic bolus CT. Radiology 1992;182(2):545-8.

[13] Matsui O, Kobayashi S, Gabata T, et al. Liver: focal hepatic mass lesion. In: Haaga JR, Dogra VS, Forsting M, et al. eds. CT \& MRI of whole body. $5^{\text {th }}$ edn. Mosby Inc., 2009; p. 1526.

[14] Baron RL, Oliver JH, Dodd GD, et al. Hepatocellular carcinoma: evaluation with biphasic, contrastenhanced, helical CT. Radiology 1996;199(2):505-11.

[15] Honda H, Ochiai K, Adachi E, et al. Hepatocellular carcinoma: correlation of CT, angiographic and histopathologic findings. Radiology 1993;189(3):85762.

[16] George RA, Godora SC, Dhagat P, et al. Computed tomographic findings in 50 cases of gallbladder carcinoma. MJAFI 2007;63(3):215-9.

[17] Kumar S, Jain A, Jain S. Gallbladder carcinoma: experience of 116 cases. Trop Gastroenterology 2001;22:172-3.

[18] Valerie V, Van Beers BE, Jean-Francois F, et al. Intrahepatic cholangiocarcinoma: MRI and pathologic correlation in 14 patients. J Computer Assisted Tomography 1997;21(1):59-65. 
[19] Asayama Y, Yoshimitsu K, Irie H, et al. Delayed phase dynamic CT enhancement as a prognostic factor for mass-forming intrahepatic cholangiocarcinoma. Radiology 2006;238(1):150-5.
[20] Foley WD, Mallisee TA, Hohenwalter MD. Multiphase hepatic CT with a multirow detector CT scanner. Am J Roentgenol 2000;175(3):679-85.

[21] Sica GT, Ji H, Ros PR. CT and MR imaging of hepatic metastases. AJR 2000;174(3):691-8. 